\title{
Significant association between long non-coding RNA HOTAIR polymorphisms and cancer susceptibility: a meta-analysis
}

This article was published in the following Dove Press journal:

OncoTargets and Therapy

I June 2016

Number of times this article has been viewed

\author{
Jian Zhang' \\ $\mathrm{Xu} \mathrm{Liu}{ }^{2}$ \\ Liang-Hao You' \\ Rui-Zhi Zhou' \\ 'Department of Cell Biology, Key \\ Laboratory of Cell Biology, Ministry \\ of Public Health and Key Laboratory \\ of Medical Cell Biology, Ministry of \\ Education, China Medical University, \\ Shenyang, People's Republic of \\ China; ${ }^{2}$ Department of Neurology, \\ First Affiliated Hospital of China \\ Medical University, Shenyang, \\ People's Republic of China
}

\begin{abstract}
HOTAIR, a well-known long non-coding RNA, is involved in carcinogenesis and progression of multiple cancers. Molecular epidemiological studies suggest that HOTAIR polymorphisms may be associated with cancer susceptibility, but the results remain controversial. To derive a more precise evaluation, we performed a meta-analysis focused on the associations between HOTAIR polymorphisms and cancer risk for the first time. PubMed, Embase, China National Knowledge Infrastructure, and Wanfang databases were searched. Odds ratios (ORs) with $95 \%$ confidence interval $(\mathrm{CI})$ were applied to assess the association between HOTAIR rs920778 C $>$ T, rs4759314 A $>$ G, rs7958904 G $>$ C, and rs1899663 G $>$ T polymorphisms and cancer susceptibility. Analyses were conducted to detect heterogeneity, sensitivity, and publication bias in order to measure the robustness of our findings. Overall, 13 related studies involving 7,151 patients and 8,740 control samples were analyzed. Significant associations between the HOTAIR rs920778 polymorphism and cancer risk were observed ( $\mathrm{T}$ vs $\mathrm{C}$ : $\mathrm{OR}=1.33,95 \%$ $\mathrm{CI}=1.17-1.53$; TT vs TC $+\mathrm{CC}: \mathrm{OR}=1.55,95 \% \mathrm{CI}=1.21-2.00 ; \mathrm{TC}+\mathrm{TT}$ vs $\mathrm{CC}: \mathrm{OR}=1.33$, $95 \% \mathrm{CI}=1.11-1.59$; TT vs $\mathrm{CC}$ : $\mathrm{OR}=2.02,95 \% \mathrm{CI}=1.31-3.10)$ in the total population, as well as in subgroup analyses. For rs4759314 $\mathrm{A}>\mathrm{G}$ polymorphism, a similarly increased risk was found in the gastric cancer group. However, significant decreases in cancer risk were observed both in the overall population and colorectal cancer group for rs $7958904 \mathrm{G}>\mathrm{C}$ polymorphism. In addition, no significant association was detected between $\mathrm{rs} 1899663 \mathrm{G}>\mathrm{T}$ polymorphism and cancer susceptibility. In conclusion, our meta-analyses suggest that HOTAIR polymorphisms may be associated with the risk of cancer development.
\end{abstract}

Keywords: HOTAIR, polymorphism, cancer susceptibility

\section{Introduction}

Cancer has now become one of the main causes of morbidity and mortality worldwide. ${ }^{1}$ Overall, approximately 14.1 million new cases and 8.2 million deaths occurred in 2012, and most of them occurred in less developed countries. ${ }^{2}$ Despite recent advances in treatment of surgery, chemotherapy, and radiotherapy, the 5-year survival rate remains low in many types of cancers. ${ }^{3}$ Therefore, it is vital to identify the factors leading to cancer susceptibility. Nowadays, many molecular epidemiological studies have reported that genetic factors may play an important role in cancer development, and the genetic predisposition is receiving increasing attention. ${ }^{4,5}$

Long non-coding RNAs (lncRNAs) are defined as a new group of transcribed RNA molecules that are longer than 200 nucleotides and have no obvious protein-coding capacity. ${ }^{6,7}$ LncRNAs were previously considered as a fake transcriptional noise, ${ }^{8}$ but now accumulating evidence suggests that they are crucial players in a wide range of
Correspondence: Jian Zhang

Department of Cell Biology, Key Laboratory of Cell Biology, Ministry of Public Health and Key Laboratory of Medical Cell Biology, Ministry of Education, China Medical University, No 77 Puhe Road, Shenyang North Area, Shenyang, Liaoning I 10122 , People's Republic of China Email jiancmu@I26.com 
biological processes including cell proliferation, survival, metabolism, and differentiation. ${ }^{9-11}$ Moreover, lncRNAs exhibit unique profiles in many types of cancers, contribute to carcinogenesis and progression, and are reasonably regarded as predictors of patient outcomes. ${ }^{12-14}$

HOTAIR, a prominently focused lncRNA, was initially identified to be implicated in breast cancer and promote tumor invasiveness and metastasis in $2007 .{ }^{15}$ It has been reported that HOTAIR could interact with PRC2 and induce its relating methylation of $\mathrm{H} 3 \mathrm{~K} 27$ to reprogram chromatin organization. ${ }^{15,16}$ Recently, the overexpression of HOTAIR and significant association with poor prognosis in a variety of human cancers including liver, breast, colon, lung, stomach, and esophageal cancers has been found. ${ }^{17-21}$ All this convincing proof indicates the oncogenic role of HOTAIR in the course of several human carcinogenesis. Therefore, increasing studies have investigated the single nucleotide polymorphisms (SNPs) in the HOTAIR locus with cancer risk. However, the results were inconsistent and inconclusive. Thus, a comprehensive meta-analysis involving the related studies was performed to assess the possible association between HOTAIR polymorphisms and cancer susceptibility.

\section{Material and methods}

\section{Search strategy}

The PubMed, Embase, China National Knowledge Infrastructure, and Wanfang databases were searched to identify studies that examined the association between HOTAIR polymorphisms and cancer susceptibility prior to January 31, 2016. The following medical subject heading terms were used: (HOTAIR OR HOX transcript antisense RNA OR long noncoding RNA OR lncRNA OR lincRNA) AND (cancer OR carcinoma OR tumor OR neoplasia OR neoplasm) AND (polymorphism OR genotype OR allele OR variant OR SNP).

\section{Eligibility criteria}

All selected studies had to meet the following criteria: 1) published studies based on case-control design assessing the association between the HOTAIR polymorphisms and cancer susceptibility; 2) the study included sufficient genotype distribution data to calculate odds ratios (ORs) and $95 \%$ confidence intervals (CIs). Studies were excluded if they investigated the progression, severity, phenotype modification, response to treatment, survival or family based studies. Moreover, meeting abstracts, case reports, editorials, and review articles were also excluded. For duplicate publications, the one with more complete design or larger sample size was finally selected.

\section{Data extraction}

Two independent researchers extracted the data from each relevant study including the first author, publication year, study country/region, ethnicity of participants (such as Asian or Caucasian), sources of controls, genotyping method, casecontrol matched status, type of cancers, Hardy-Weinberg equilibrium (HWE) status of controls, and number of genotypes in cancer cases and controls. Disagreements were reconciled through group discussion. The HWE was calculated based on the genotypes of the controls.

\section{Statistical analysis}

ORs with $95 \%$ CIs were used to assess the strength of the association between the HOTAIR polymorphisms and cancer risk. For the HOTAIR rs920778 $\mathrm{C}>\mathrm{T}$ polymorphism, the pooled ORs were obtained for allele ( $\mathrm{T}$ vs $\mathrm{C}$ ), recessive (TT vs TC + CC), dominant (TC + TT vs CC), and homozygous (co-dominant) model (TT vs CC). Similar genetic models were also assessed for HOTAIR rs4759314 A $>$ G, rs7958904 $\mathrm{G}>\mathrm{C}$, and rs1899663 G>T variants. Subgroup analyses were performed based on ethnicity, source of controls, genotyping methods, type of cancers, case-control matched status, and HWE status of controls. ORs were calculated using the random-effect model when the $I^{2}$ was greater than $50 \%$. Otherwise, a fixed-effect model was adopted. In order to evaluate the stability of the results, sensitivity analysis was used, which meant omitting one study at a time, and then compared to show whether a significant difference existed between the former and the latter results. Publication bias was examined by the visual inspection of funnel plot, and Egger's regression test. Data were analyzed and processed using Stata 12.0 (StataCorp LP, College Station, TX, USA). $P<0.05$ was considered statistically significant.

\section{Results Study characteristics}

A systematic search of the literature identified 135 relevant studies. The study selection process is shown in Figure 1. Following the selection criteria, 127 studies were excluded from our research due to various deficiencies. Ultimately, eight eligible articles were selected with adequate data, including eight studies on rs920778 C $>$ T, ${ }^{22-26}$ eight studies on rs4759314 $\mathrm{A}>\mathrm{G},{ }^{24-29}$ three publications on rs1899663 $\mathrm{G}>\mathrm{T},{ }^{24-26}$ and three studies on $\mathrm{rs} 7958904 \mathrm{G}>\mathrm{C},{ }^{28,29}$ respectively. All studies were published between 2014 and 2016. Only two studies involved Caucasian populations, and other studies involved Asian populations. The genotype distribution was in agreement with HWE in all studies except for one study 


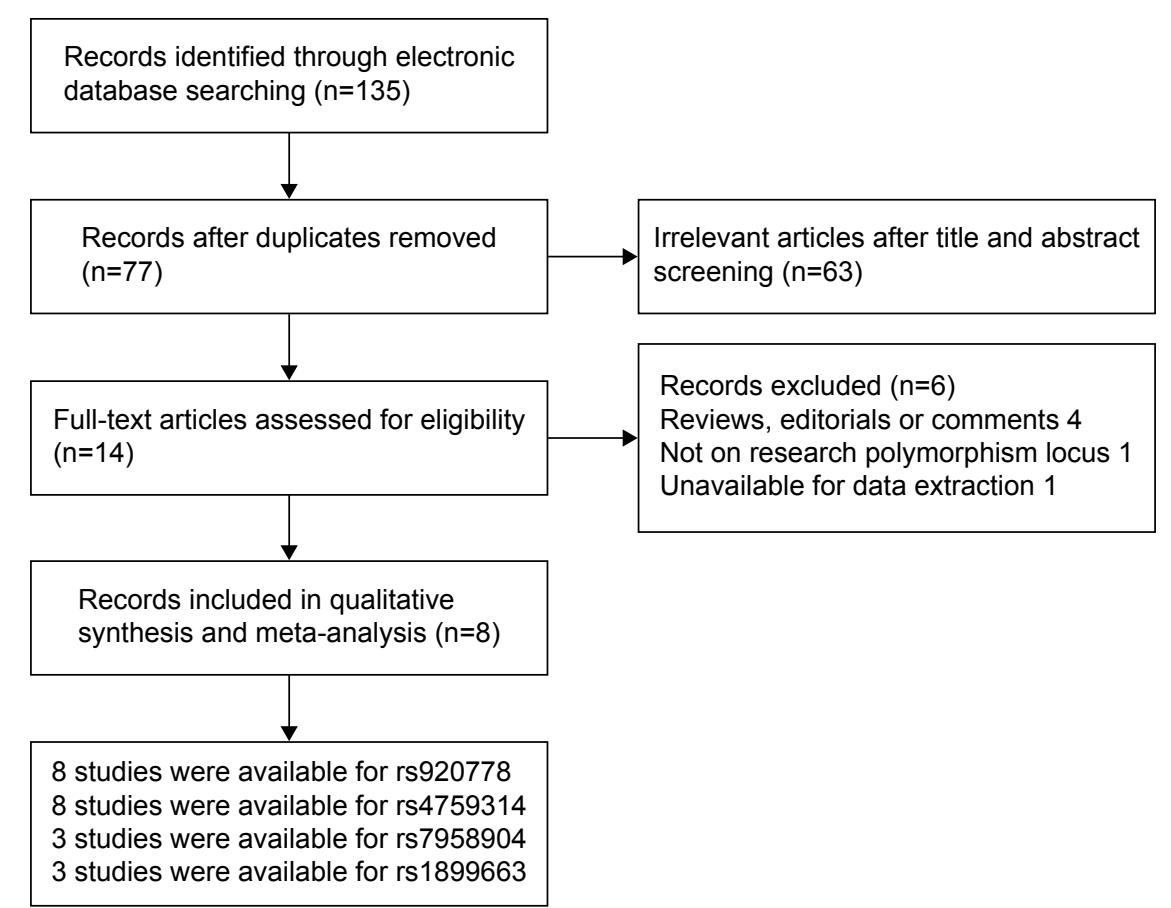

Figure I Flow diagram of the study selection process.

of HOTAIR rs920778 C > T polymorphism. Detailed characteristics of studies included are summarized in Table 1.

\section{Association between the HOTAIR rs920778 C $>$ T polymorphism and cancer risk}

A total of eight relevant studies, consisting of 3,627 patients and 4,585 controls, were examined for the association between the HOTAIR rs $920778 \mathrm{C}>\mathrm{T}$ polymorphism and cancer risk. The combined analyses revealed a significantly increased risk of cancer for this polymorphism in all four genetic models ( $\mathrm{T}$ vs $\mathrm{C}$ : $\mathrm{OR}=1.33,95 \% \mathrm{CI}=1.17-1.53, P<0.01$, $P^{2}=68.1 \%$; TT vs TC $+\mathrm{CC}: \mathrm{OR}=1.55,95 \% \mathrm{CI}=1.21-2.00$, $P=0.001, I^{2}=59.7 \%$; TC + TT vs CC: $\mathrm{OR}=1.33,95 \%$ $\mathrm{CI}=1.11-1.59, P=0.002, P^{2}=63.6 \%$; TT vs $\mathrm{CC}: \mathrm{OR}=2.01$, $95 \% \mathrm{CI}=1.31-3.10, P=0.001, P=77.0 \%$; Figure 2, Table 2 ).

Subsequent analyses accounting for ethnicity revealed similar results in Asian populations, using all four genotype models. Significant correlations with increased cancer risk were also observed in the population-based control group and studies restricted to HWE. Enhanced cancer risk was also observed in the subgroup analysis with genotyping method of restriction fragment length polymorphism under all four genetic models. Moreover, elevated risks of gastric cancer (T vs C: $\mathrm{OR}=1.32,95 \% \mathrm{CI}=1.01-1.72, P=0.045, P^{2}=73.8 \%$; TC + TT vs CC: $\mathrm{OR}=1.36,95 \% \mathrm{CI}=1.02-1.83, P=0.039$,
$I^{2}=59.0 \%$; TT vs CC: $\mathrm{OR}=2.12,95 \% \mathrm{CI}=1.00-4.51$, $P=0.050, I^{2}=78.1 \%$ ) and esophageal cancer ( $\mathrm{T}$ vs $\mathrm{C}: \mathrm{OR}=1.46$, 95\% CI $=1.32-1.61, P<0.001, I^{2}=0$; TT vs TC $+\mathrm{CC}$ : $\mathrm{OR}=1.96,95 \% \mathrm{CI}=1.48-2.59, P<0.001, P^{2}=59.7 \%$; $\mathrm{TC}+\mathrm{TT}$ vs CC: $\mathrm{OR}=1.44,95 \% \mathrm{CI}=1.27-1.62, P<0.001, I^{2}=0$; TT vs $\mathrm{CC}$ : $\mathrm{OR}=2.81,95 \% \mathrm{CI}=2.13-3.71, P<0.001, I^{2}=2.2 \%$; Table 2) were detected.

Sensitivity analysis showed that no single study qualitatively changed the pooled ORs with corresponding 95\% CI, indicating that the results of this meta-analysis were highly stable (see Figure 3 for allele contrast model). Visual inspection of funnel plot did not reveal any asymmetrical evidence (see Figure 4 for allele contrast model). The results were further supported by the analysis of the data with Egger's test (T vs $C: P=0.121$; TT vs TC + CC: $P=0.062 ; \mathrm{TC}+\mathrm{TT}$ vs $C \mathrm{C}$ : $P=0.243$; TT vs CC: $P=0.195$ ).

\section{Association between HOTAIR rs47593।4 $A>G$ polymorphism and cancer risk}

Eight studies consisting of 5,526 cases and 6,659 controls were included in the analysis to determine whether the HOTAIR rs4759314 A $>$ G polymorphism was associated with cancer risk. Overall, no significant association was observed in all four models (Table 2). Only two genetic models (for $\mathrm{G}$ vs $\mathrm{A}, \mathrm{OR}=1.29,95 \% \mathrm{CI}=1.10-1.51, P=0.002$, $P^{2}=43.5 \%$; for $\mathrm{GA}+\mathrm{GG}$ vs AA, $\mathrm{OR}=1.32,95 \% \mathrm{CI}=1.12-1.56$, 


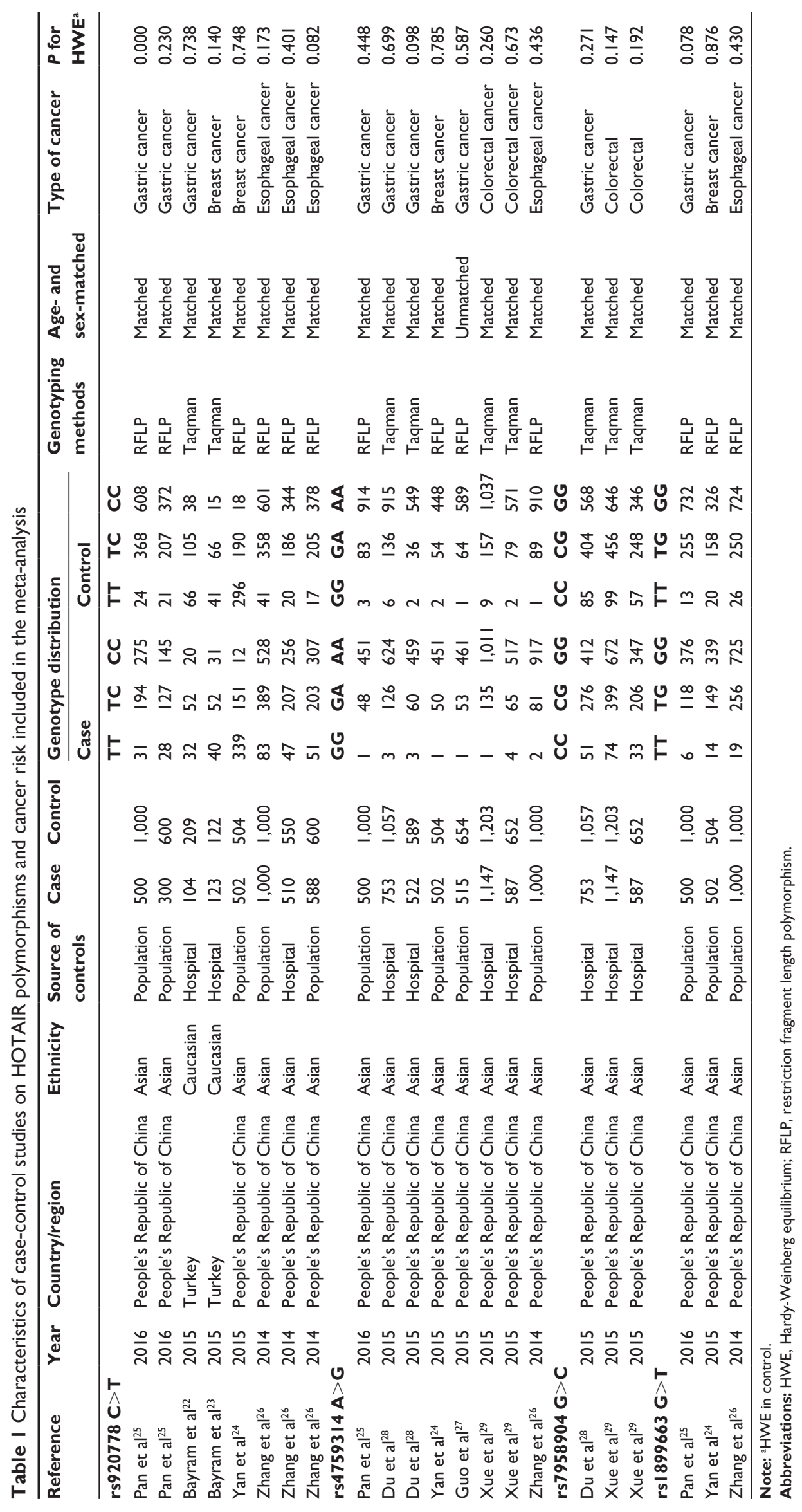




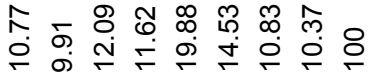

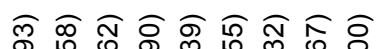

$\widehat{\bar{u}}$ ले กิ

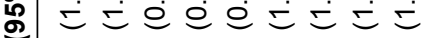

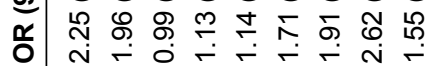

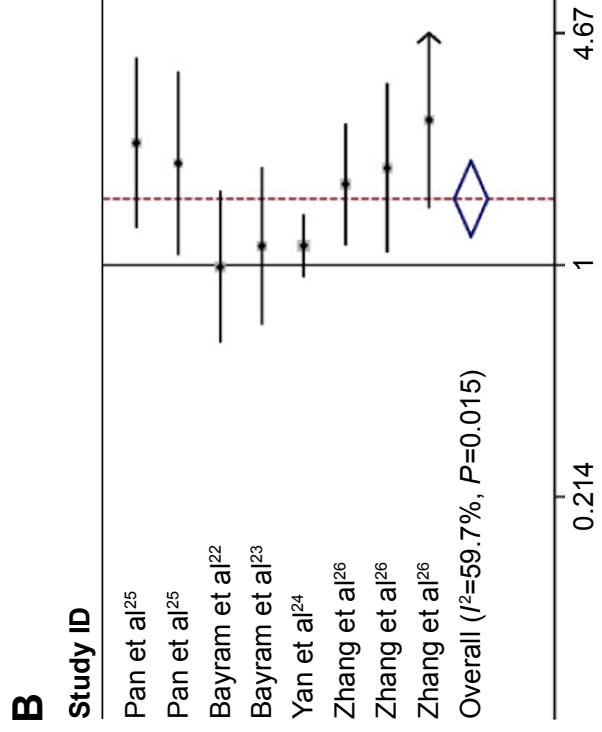

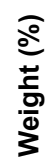

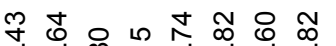

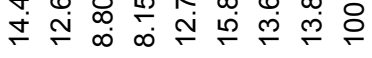

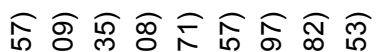

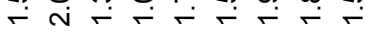

号

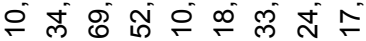

边 E $=$ o $=5=5=$

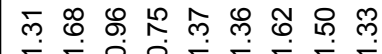

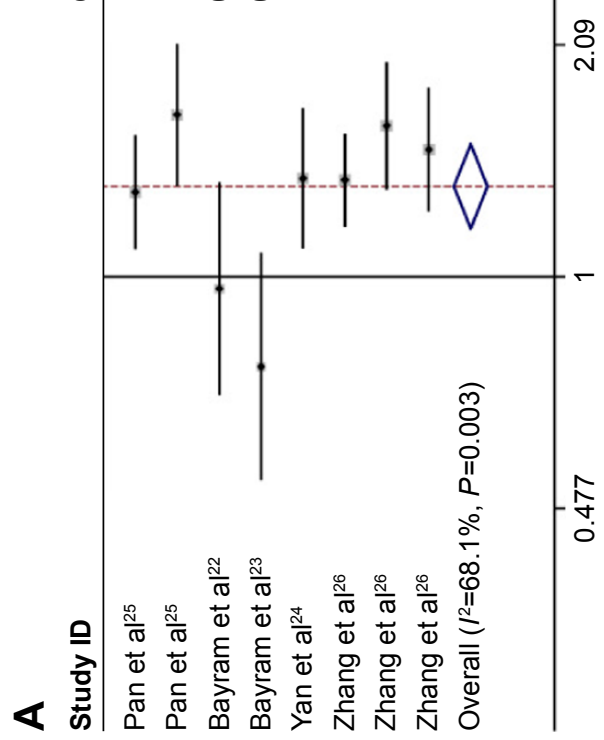

产|

8 용 \& 8 용ㅇㅇ

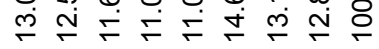

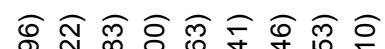

पै + ن

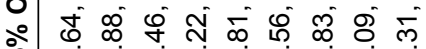

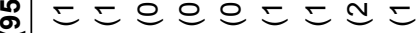

范
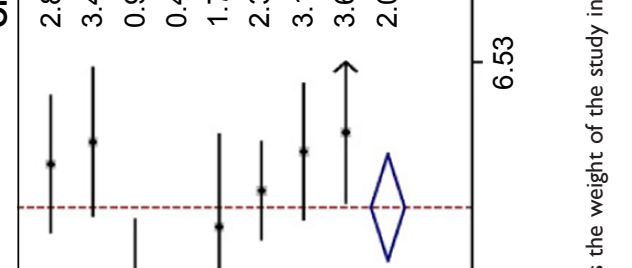

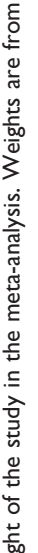

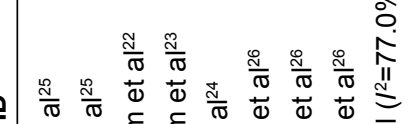

วิ

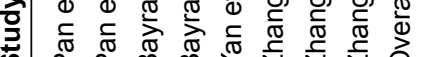

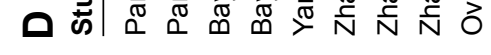

혼

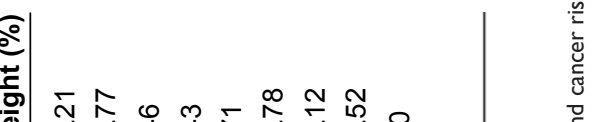

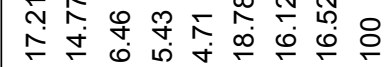

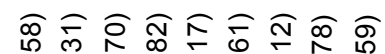

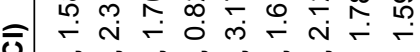

ㅇำ

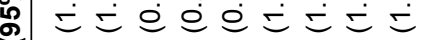

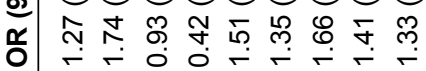

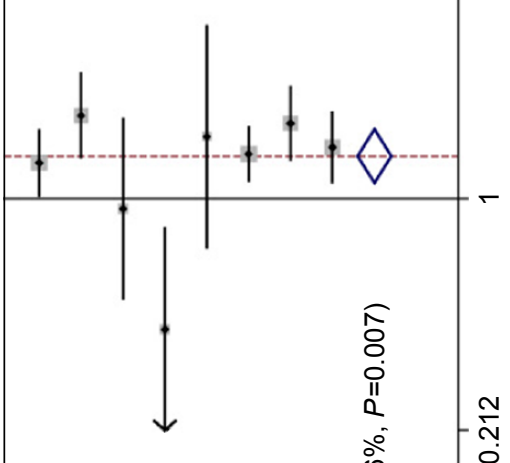

ष्ष

造 일

ㅎํㄹ 


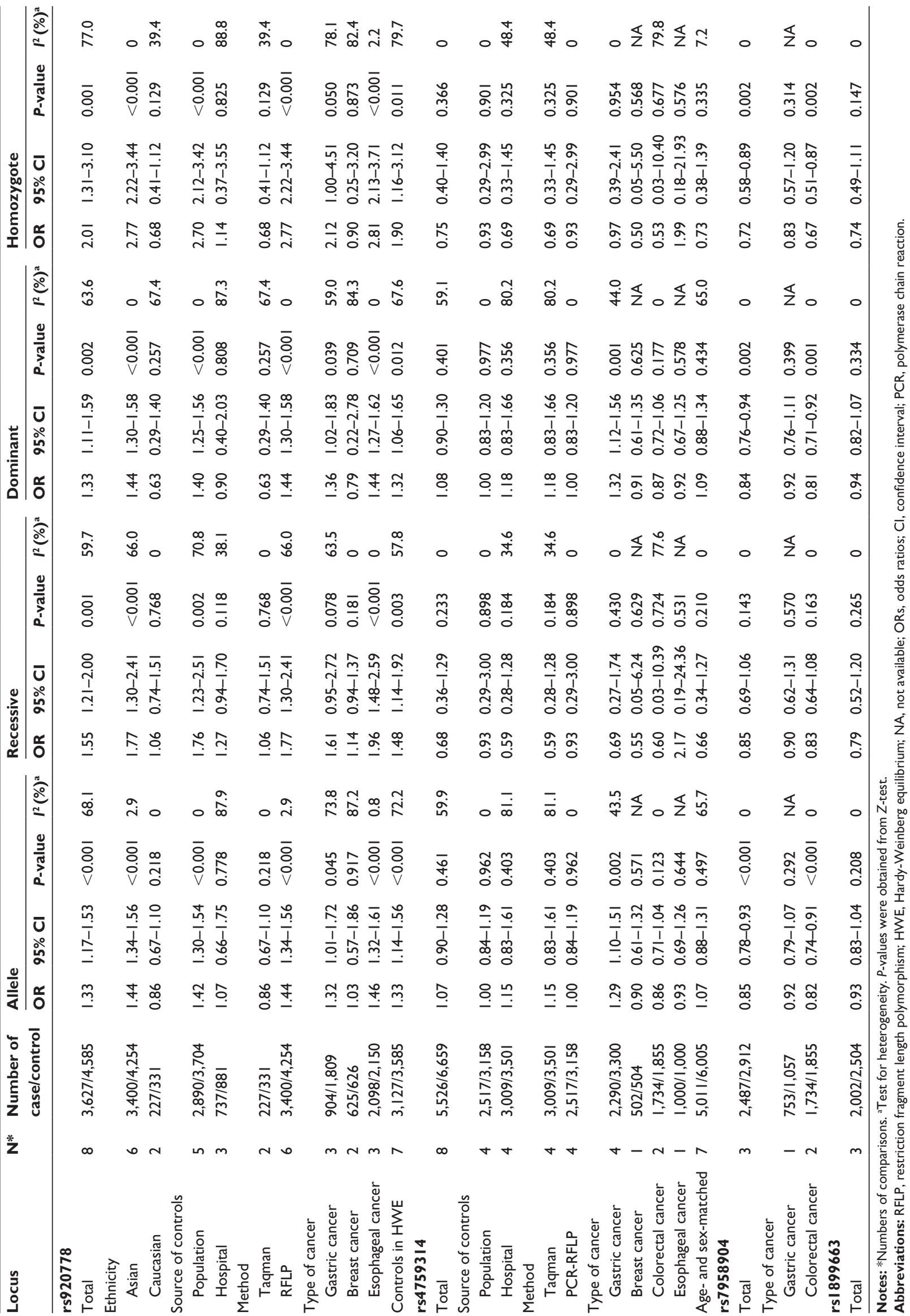




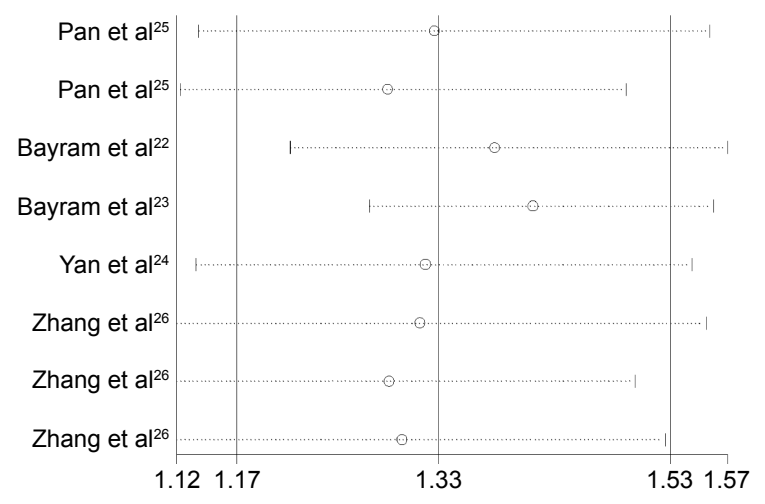

Figure 3 Sensitivity analysis via deletion of each individual study reflects the relative influence of each individual dataset on the pooled ORs in the allele contrast model of HOTAIR rs 920778 polymorphism.

Abbreviation: ORs, odds ratios.

$\left.P=0.001, I^{2}=44.0 \%\right)$ revealed increased risk in the gastric cancer group. Further subgroup analyses of genotyping method, source of controls, and case-control matched status were conducted, and no significant association was identified (Table 2). The pooled ORs did not exhibit any change with sensitivity analysis, and no publication bias was observed (G vs A: $P=0.350 ; \mathrm{GG}$ vs GA + AA: $P=0.902 ; \mathrm{GA}+\mathrm{GG}$ vs AA: $P=0.408$; GG vs AA: $P=0.823$ ).

\section{Association between HOTAIR rs7958904 $\mathrm{G}>\mathrm{C}$ polymorphism and cancer risk}

Three eligible studies including 2,487 cases and 2,912 controls focused on the association of HOTAIR rs $7958904 \mathrm{G}>\mathrm{C}$ polymorphism with cancer. The heterogeneity among studies, measured by $I^{2}$ statistic, was not significant in all genetic models $\left(I^{2}<0.5\right)$. Therefore, the fixed effect model was used in all genetic models. A significant decrease in cancer risk

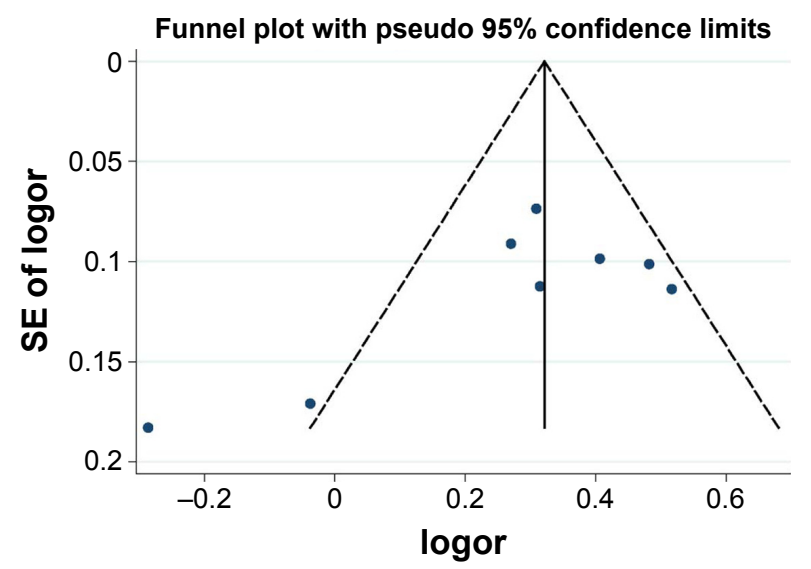

Figure 4 Funnel plot analysis to detect publication bias for the allele contrast model of HOTAIR rs920778 polymorphism.

Abbreviation: SE, standard error. was observed in the overall population $(\mathrm{C}$ vs $\mathrm{G}$ : $\mathrm{OR}=0.85$, 95\% CI $=0.78-0.93, P<0.001, I^{2}=0 \%$; $\mathrm{CG}+\mathrm{CC}$ vs GG: $\mathrm{OR}=0.84,95 \% \mathrm{CI}=0.76-0.94, P=0.002, I^{2}=0 ; \mathrm{CC}$ vs GG: $\mathrm{OR}=0.72,95 \% \mathrm{CI}=0.58-0.89, P=0.002, I^{2}=0$; Table 2 ). Moreover, these results were consistent with subgroup analysis of the colorectal cancer group (Table 2). Sensitivity analysis was conducted, and no conspicuous change of the pooled ORs was detected. No publication bias was observed, indicating that the results are statistically robust $(\mathrm{C}$ vs $\mathrm{G}$ : $P=0.757 ; \mathrm{CC}$ vs $\mathrm{CG}+\mathrm{GG}: P=0.354 ; \mathrm{CG}+\mathrm{CC}$ vs $\mathrm{GG}$ $P=0.870$; CC vs GG: $P=0.587)$.

\section{Association between the HOTAIR rs I899663 G > T polymorphism and cancer risk}

Three studies with 2,002 cases and 2,504 controls were included in the HOTAIR rs $1899663 \mathrm{G}>$ T polymorphism and cancer risk research. No significant associations were found in all four models for this SNP locus (Table 2).

\section{Discussion}

Genetic variants, mainly composed of SNPs, have been shown to influence the susceptibility of patients to cancer and have attracted increasing attention. LncRNAs play crucial roles in a wide range of biological processes and are involved in the development and progression of multiple cancers. SNPs in several lncRNAs previously identified to be involved in carcinogenesis have been reported to be associated with cancer risk. ${ }^{30,31}$

Recently, several molecular epidemiological studies have been conducted to evaluate the association between polymorphisms of HOTAIR and the risk of cancer development, but results have remained conflicting. Regarding the HOTAIR rs920778 $\mathrm{C}>\mathrm{T}$ polymorphism, Pan et al reported that the TT carriers had a 1.66- and 1.87-fold increased gastric cancer risk in Jinan and Huaian populations of People's Republic of China compared with the $\mathrm{CC}$ carriers. ${ }^{25} \mathrm{~A}$ similar increase in esophageal cancer risk was also observed in three independent case-control sets consisting of 4,248 Chinese subjects. ${ }^{26}$ However, CC genotype of HOTAIR rs920778 polymorphism was found to significantly increase the risk of breast cancer in a Turkish population, whereas another study demonstrated that the HOTAIR rs920778 polymorphism had not played any major role in genetic susceptibility to gastric carcinogenesis. ${ }^{22,23}$

To our knowledge, this is the first meta-analysis to date focused on the association between polymorphisms in 
lncRNA HOTAIR and cancer susceptibility specially. All the relative studies about cancer risk and four polymorphisms of HOTAIR were collected to make a precise conclusion. Overall, significant increased risk of cancer was observed for the HOTAIR rs920778 C > T polymorphism. In subgroup analyses by ethnicity, we found that individuals with the $\mathrm{T}$ allele and mutated genotypes had a significant increased cancer risk in Asian populations, suggesting that the increased cancer risk may be ethno-specific. Furthermore, it is worth noting that some significantly increased risks were observed in gastric and esophageal cancer, but not the breast cancer group. Similarly, the result that $\mathrm{rs} 4759314 \mathrm{~A}>\mathrm{G}$ polymorphism just increased the risk of gastric cancer rather than other types of cancers was revealed in our meta-analysis. While significant decreases in cancer risk were observed both in overall population and colorectal cancer group, indicating that rs7958904 $\mathrm{G}>\mathrm{C}$ polymorphism might be a protective factor especially for colorectal cancer. In addition, a negative correlation was observed in the rs $1899663 \mathrm{G}>\mathrm{T}$ polymorphism analysis.

Although the number of the included studies was relatively small, we believe that the findings can help to explain the association. First, in the sensitivity analysis, no significant changes were found after omitting each study at a time, indicating the relative stability and credibility of the results. Second, the genotype distributions in the controls of four selected SNP loci were all mostly consistent with HWE except for one study in rs920778 polymorphism analysis. Third, the visual inspection of funnel plot and Egger's test proved that almost no apparent publication bias existed in our meta-analysis. All these would guarantee the reliability of results.

However, it is important to note the limitations of our meta-analysis. First, heterogeneity across studies existed for rs920778 and rs4759314 polymorphisms. Unfortunately, we did not perform meta-regression analysis which is not suitable for assessing heterogeneity with a sample size less than ten. ${ }^{32}$ Considering that ethnic diversity, study design difference, and measurement error may contribute to common sources of heterogeneity, ${ }^{33}$ we performed subgroup analyses to explore the source of heterogeneity. For rs920778, the results of subgroup analyses did not effectively eliminate the heterogeneity, indicating that all above factors should be taken into consideration. Nevertheless, subgroup analyses were successfully used to relieve moderate heterogeneity bias in the rs4759314 polymorphism analysis within the population-based control group and the genotyping method of restriction fragment length polymorphism group, suggesting that control sources and genotyping method may influence heterogeneity. The second limitation lies in the ethnicity of the subjects. Most of the patients were Asians in the present study and this limited the general application of the results to other populations. Third, all of our results may be influenced by casualness due to the small number of studies included and the limited sample size of each study. Finally, cancer is a multi-factorial malignant disease that likely arises from complex interactions between genetic mutations, environmental changes, lifestyle, diet, age, and sex. In our meta-analysis, we only focused on the HOTAIR polymorphisms, while the fundamental underlying mechanisms cannot be explained clearly due to unadjusted databases.

\section{Conclusion}

In conclusion, the current meta-analysis indicated that three functional polymorphisms of HOTAIR rs920778 C $>$ T, rs4759314 $\mathrm{A}>\mathrm{G}$, and rs7958904 $\mathrm{G}>\mathrm{C}$ may play an important role in the development of cancer. Given the limitations in the present meta-analysis, the results need to be interpreted with caution. Large-scale, case-control studies with rigorous designs should be conducted to confirm the association of above functional polymorphisms in lncRNA HOTAIR and cancer risk in the future.

\section{Acknowledgments}

This work was supported by the National Natural Science Foundation of China (grant number 81302127, 81400950) and the Joint Specialized Research Fund for the Doctoral Program of Higher Education in China (grant number 20132104120018).

\section{Disclosure}

The authors report no conflicts of interest in this work.

\section{References}

1. Bray F, Ren JS, Masuyer E, Ferlay J. Global estimates of cancer prevalence for 27 sites in the adult population in 2008. Int J Cancer. 2013; 132(5):1133-1145.

2. Ferlay J, Soerjomataram I, Dikshit R, et al. Cancer incidence and mortality worldwide: sources, methods and major patterns in GLOBOCAN 2012. Int J Cancer. 2015;136(5):E359-E386.

3. Gonzalez CA, Agudo A. Carcinogenesis, prevention and early detection of gastric cancer: where we are and where we should go. Int $J$ Cancer. 2012;130(4):745-753.

4. Zucman-Rossi J, Villanueva A, Nault JC, Llovet JM. Genetic Landscape and Biomarkers of Hepatocellular Carcinoma. Gastroenterology. 2015;149(5):1226-1239.e4.

5. Mocellin S, Verdi D, Pooley KA, Nitti D. Genetic variation and gastric cancer risk: a field synopsis and meta-analysis. Gut. 2015;64(8):1209-1219.

6. Derrien T, Johnson R, Bussotti G, et al. The GENCODE v7 catalog of human long noncoding RNAs: analysis of their gene structure, evolution, and expression. Genome Res. 2012;22(9):1775-1789.

7. Shi X, Sun M, Liu H, Yao Y, Song Y. Long non-coding RNAs: a new frontier in the study of human diseases. Cancer Lett. 2013;339(2):159-166. 
8. Guttman M, Donaghey J, Carey BW, et al. lincRNAs act in the circuitry controlling pluripotency and differentiation. Nature. 2011;477(7364): 295-300.

9. Struhl K. Transcriptional noise and the fidelity of initiation by RNA polymerase II. Nat Struct Mol Biol. 2007;14(2):103-105.

10. Ebisuya M, Yamamoto T, Nakajima M, Nishida E. Ripples from neighbouring transcription. Nat Cell Biol. 2008;10(9):1106-1113.

11. Mercer TR, Dinger ME, Mattick JS. Long non-coding RNAs: insights into functions. Nat Rev Genet. 2009;10(3):155-159.

12. Gibb EA, Brown CJ, Lam WL. The functional role of long non-coding RNA in human carcinomas. Mol Cancer. 2011;10:38.

13. Wapinski O, Chang HY. Long noncoding RNAs and human disease. Trends Cell Biol. 2011;21(6):354-361.

14. Deng Q, He B, Gao T, et al. Up-regulation of $91 \mathrm{H}$ promotes tumor metastasis and predicts poor prognosis for patients with colorectal cancer. PloS One. 2014;9(7):e103022.

15. Gupta RA, Shah N, Wang KC, et al. Long non-coding RNA HOTAIR reprograms chromatin state to promote cancer metastasis. Nature. 2010;464(7291):1071-1076.

16. Rinn JL, Kertesz M, Wang JK, et al. Functional demarcation of active and silent chromatin domains in human HOX loci by noncoding RNAs. Cell. 2007;129(7):1311-1323.

17. Kogo R, Shimamura T, Mimori K, et al. Long noncoding RNA HOTAIR regulates polycomb-dependent chromatin modification and is associated with poor prognosis in colorectal cancers. Cancer Res. 2011;71(20): 6320-6326.

18. Yang Z, Zhou L, Wu LM, et al. Overexpression of long non-coding RNA HOTAIR predicts tumor recurrence in hepatocellular carcinoma patients following liver transplantation. Ann Surg Oncol. 2011; 18(5):1243-1250.

19. Lu L, Zhu G, Zhang C, et al. Association of large noncoding RNA HOTAIR expression and its downstream intergenic $\mathrm{CpG}$ island methylation with survival in breast cancer. Breast Cancer Res Treat. 2012 136(3):875-883.

20. Nakagawa T, Endo H, Yokoyama M, et al. Large noncoding RNA HOTAIR enhances aggressive biological behavior and is associated with short disease-free survival in human non-small cell lung cancer. Biochem Biophys Res Commun. 2013;436(2):319-324.

21. Lv XB, Lian GY, Wang HR, Song E, Yao H, Wang MH. Long noncoding RNA HOTAIR is a prognostic marker for esophageal squamous cell carcinoma progression and survival. PloS One. 2013;8(5):e63516.
22. Bayram S, Ulger Y, Sumbul AT, et al. A functional HOTAIR rs 920778 polymorphism does not contributes to gastric cancer in a Turkish population: a case-control study. Fam Cancer. 2015;14(4):561-567.

23. Bayram S, Sumbul AT, Batmaci CY, Genc A. Effect of HOTAIR rs920778 polymorphism on breast cancer susceptibility and clinicopathologic features in a Turkish population. Tumour Biol. 2015; 36(5):3863-3870.

24. Yan R, Cao J, Song C, et al. Polymorphisms in lncRNA HOTAIR and susceptibility to breast cancer in a Chinese population. Cancer Epidemiol. 2015;39(6):978-985.

25. Pan W, Liu L, Wei J, et al. A functional lncRNA HOTAIR genetic variant contributes to gastric cancer susceptibility. Mol Carcinog. 2016; 55(1):90-96.

26. Zhang X, Zhou L, Fu G, et al. The identification of an ESCC susceptibility SNP rs920778 that regulates the expression of $\operatorname{lncRNA}$ HOTAIR via a novel intronic enhancer. Carcinogenesis. 2014;35(9):2062-2067.

27. Guo W, Dong Z, Bai Y, et al. Associations between polymorphisms of HOTAIR and risk of gastric cardia adenocarcinoma in a population of north China. Tumour Biol. 2015;36(4):2845-2854.

28. Du M, Wang $\mathrm{W}$, Jin $\mathrm{H}$, et al. The association analysis of lncRNA HOTAIR genetic variants and gastric cancer risk in a Chinese population. Oncotarget. 2015;6(31):31255-31262.

29. Xue Y, Gu D, Ma G, et al. Genetic variants in lncRNA HOTAIR are associated with risk of colorectal cancer. Mutagenesis. 2015;30(2):303-310.

30. Xue Y, Wang M, Kang M, et al. Association between Incrna PCGEM1 polymorphisms and prostate cancer risk. Prostate Cancer Prostatic Dis. 2013;16(2):139-144, S131.

31. Liu Y, Pan S, Liu L, et al. A genetic variant in long non-coding RNA HULC contributes to risk of HBV-related hepatocellular carcinoma in a Chinese population. PloS One. 2012;7(4):e35145.

32. Schmid CH, Stark PC, Berlin JA, Landais P, Lau J. Meta-regression detected associations between heterogeneous treatment effects and study-level, but not patient-level, factors. J Clin Epidemiol. 2004;57(7): 683-697.

33. Thompson SG. Why sources of heterogeneity in meta-analysis should be investigated. BMJ. 1994;309(6965):1351-1355.
OncoTargets and Therapy

\section{Publish your work in this journal}

OncoTargets and Therapy is an international, peer-reviewed, open access journal focusing on the pathological basis of all cancers, potential targets for therapy and treatment protocols employed to improve the management of cancer patients. The journal also focuses on the impact of management programs and new therapeutic agents and protocols on

\section{Dovepress}

patient perspectives such as quality of life, adherence and satisfaction. The manuscript management system is completely online and includes a very quick and fair peer-review system, which is all easy to use. Visit http://www.dovepress.com/testimonials.php to read real quotes from published authors. 